\title{
Spectra of modular and small-world matrices
}

\author{
Reimer Kühn $^{1}$ and Jort van Mourik ${ }^{2}$ \\ ${ }^{1}$ Mathematics Department, King's College London, Strand, London WC2R 2LS, UK \\ 2 NCRG, Aston University, Aston Triangle, Birmingham B4 7ET, UK \\ E-mail: reimer.kuehn@kcl.ac.uk
}

Received 7 December 2010, in final form 28 February 2011

Published 28 March 2011

Online at stacks.iop.org/JPhysA/44/165205

\begin{abstract}
We compute spectra of symmetric random matrices describing graphs with general modular structure and arbitrary inter- and intra-module degree distributions, subject only to the constraint of finite mean connectivities. We also evaluate spectra of a certain class of small-world matrices generated from random graphs by introducing shortcuts via additional random connectivity components. Both adjacency matrices and the associated graph Laplacians are investigated. For the Laplacians, we find Lifshitz-type singular behaviour of the spectral density in a localized region of small $|\lambda|$ values. In the case of modular networks, we can identify contributions of local densities of state from individual modules. For small-world networks, we find that the introduction of short cuts can lead to the creation of satellite bands outside the central band of extended states, exhibiting only localized states in the band gaps. Results for the ensemble in the thermodynamic limit are in excellent agreement with those obtained via a cavity approach for large finite single instances, and with direct diagonalization results.
\end{abstract}

PACS numbers: 02.50.-r, 05.10.-a

(Some figures in this article are in colour only in the electronic version)

\section{Introduction}

The past decade has seen a considerable activity in the study of random graphs (see, e.g., [1] or [2-4] for recent reviews), as well as concurrent intensive studies in spectral properties of sparse random matrices $[2,5-8]$, the latter providing one of the key tools to study properties of the former. Moments of the spectral density of an adjacency matrix describing a graph, for instance, give complete information about the number of walks returning to the originating vertex after a given number of steps, thus containing information about local topological properties of such graphs. Spectral properties, specifically properties of eigenvectors corresponding to the largest eigenvalue of the modularity matrices of a graph and of its subgraphs-matrices closely related to the corresponding adjacency matrices—can 
be used for efficient modularity and community detection in networks [9], and so on. Much of this activity has been motivated by the fact that a large number of systems, natural and artificial, can be described using network descriptions of underlying interaction patterns, and the language and tools of graph theory and random matrix theory for their quantitative analysis.

Though the study of spectral properties of sparse symmetric matrices was initiated by Bray and Rodgers already in the late 1980s [10,11], fairly complete analytic and numerical control over the problem has emerged only recently $[12,13]$, effectively using generalizations of earlier ideas developed by Abou-Chacra et al [14] for Bethe lattices. Analytical results for spectral properties of sparse matrices had typically been based either on the single defect or effective medium approximations (SDA, EMA) [5, 15-17] or were restricted to the limit of large average connectivity $[18,19]$. Alternatively, spectra for systems with heterogeneity induced by scale-free or small-world connectivity $[8,20]$ or as a result of an explicitly modular structure [21] were obtained through numerical diagonalization. Analytical results for spectra of modular systems [22] and for systems with topological constraints beyond degree distributions [23] are still very recent.

The purpose of this paper is to expand the scope of [22] in two ways: (i) by providing spectra of random matrices describing graphs with general modular structure and arbitrary inter- and intra-module degree distributions, subject only to the constraint of finite mean connectivities, and (ii) by computing spectra for a class of small-world systems, constructed as regular random graphs with an additional connectivity component providing long-range interactions and thus shortcuts. The connection between these two seemingly different problems is mainly provided by the close similarity of the methods used to study these systems.

Our study is motivated by the fact that modularity of systems, and thus networks of interactions are a natural property of large structured systems; think of compartmentalization in multi-cellular organisms, sub-structures and organelles inside cells and the induced structures, e.g. in protein-protein interaction networks, or think of large corporates with several subdivisions, to name but a few examples.

In section 2.1 we introduce the type of multi-modular system and the associated random matrices we are going to study. A replica analysis of the problem is described in section 2.2, with (replica-symmetric) self-consistency equations formulated in section 2.3, but the bulk of the derivation relegated to the appendix. Section 3 introduces a class of small-world networks generated from (regular) random graphs by introducing shortcuts via a second, longrange connectivity component, and briefly describes the rather minimal modifications in the theoretical description needed to analyse those systems as well. In section 4 we present a selection of results. Our main conclusions are outlined in section 5.

\section{Modular systems}

\subsection{Multi-modular systems and associated random matrices}

We consider a system of size $N$ which consists of $m$ modules $\mathcal{N}_{\mu}, \mu=1, \ldots, m$. We use $N_{\mu}$ to denote the size of the module $\mathcal{N}_{\mu}$ and assume that each module occupies a finite fraction of the entire system, $N_{\mu}=f_{\mu} N$, with $f_{\mu}>0$ for all $\mu$ and

$$
\sum_{\mu=1}^{m} f_{\mu}=1
$$

Details of the modular structure are encoded in the $N \times N$ connectivity matrix $c=\left(c_{i j}\right)$, whose matrix elements $c_{i j}$ describe whether a link between nodes $i$ and $j$ exists $\left(c_{i j}=1\right)$ or 
not $\left(c_{i j}=0\right)$. To each site $i$ of the system, we assign a connectivity vector $\boldsymbol{k}_{i}=\left(k_{i}^{v}\right)$, whose components

$$
k_{i}^{v}=\sum_{j \in \mathcal{N}_{v}} c_{i j}
$$

give the number of connections between site $i$ and (other) sites in module $v$. The $\boldsymbol{k}_{i}$ are taken to be fixed according to some given distribution which we assume to depend only on the module to which $i$ belongs, and which has finite means

$$
\left\langle k_{i}^{v}\right\rangle_{\mu}=c^{\mu \nu}, \quad \mathrm{i} \in \mathcal{N}_{\mu}
$$

for the components, but is otherwise arbitrary. We use $\langle\ldots\rangle_{\mu}$ to denote an average over the distribution of coordinations for vertices in module $\mathcal{N}_{\mu}$. Consistency required by symmetry entails $c^{\mu \nu} / N_{\nu}=c^{\nu \mu} / N_{\mu}$ or alternatively $f_{\mu} c^{\mu \nu}=f_{\nu} c^{\nu \mu}$.

We note in passing that it is possible to include extensive intra-module and inter-module connections in addition to the finite connectivity structure described above as in [22], but we have decided not to do so here. Also we point out that in the modular graphs considered here, the vertices are always associated with irreducible degrees of freedom. Thus, in the protein example mentioned above we would associate a vertex for instance with a single unique protein species. The fact that proteins are active in, or interact with, more than one cellular component is in our formulation described by the fact that the given vertex can have interactions to vertices in several other modules. This should cover the majority of cases; further generalizations are possible by treating a given protein acting in different cell components as distinct species for each component.

Starting from the modular structure defined by the connectivity matrix $c$, we consider two types of random matrix inheriting the modular structure. The first is defined by giving random weights to the links, thereby defining random matrices $M$ of the form

$$
M_{i j}=c_{i j} K_{i j},
$$

where we assume that the statistics of the $K_{i j}$ respects the modular structure defined by $c$ in that it only depends on the modules to which $i$ and $j$ belong. The second is related to the first by introducing zero row-sum constraints, resulting in matrices of the form

$$
L_{i j}=c_{i j} K_{i j}-\delta_{i j} \sum_{k} c_{i k} K_{i k}
$$

In the special case $K_{i j}=$ const., one recovers the connectivity matrices themselves and the discrete graph Laplacians, respectively.

The spectral density of a given matrix $M$ can be computed from its resolvent via

$$
\begin{aligned}
\rho_{M}(\lambda) & =\lim _{\varepsilon \searrow 0} \frac{1}{\pi} \operatorname{Im} \operatorname{Tr}\left[\lambda_{\varepsilon} \mathbb{1}-M\right]^{-1} \\
& =\lim _{\varepsilon \searrow 0} \frac{-2}{N \pi} \operatorname{Im} \frac{\partial}{\partial \lambda} \ln \operatorname{det}\left[\lambda_{\varepsilon} \mathbb{1}-M\right]^{-1 / 2},
\end{aligned}
$$

in which $\lambda_{\varepsilon} \equiv \lambda-\mathrm{i} \varepsilon$, and the inverse square root of the determinant is obtained as a Gaussian integral. We are interested in the average spectral density obtained from (6) by taking an average over the ensemble of matrices considered, thus in

$$
\rho(\lambda)=\lim _{\varepsilon \searrow 0} \frac{-2}{N \pi} \operatorname{Im} \frac{\partial}{\partial \lambda}\left\langle\ln \left[\int \prod_{i} \frac{\mathrm{d} u_{i}}{\sqrt{2 \pi / \mathrm{i}}} \exp \left\{-\frac{\mathrm{i}}{2} \sum_{i j} u_{i}\left[\lambda_{\varepsilon} \delta_{i j}-M_{i j}\right] u_{j}\right\}\right]\right\rangle,
$$

where angled brackets on the rhs denote an average over connectivities $\left\{c_{i j}\right\}$ and weights $\left\{K_{i j}\right\}$ of the non-vanishing matrix elements. For the ensembles considered here the spectral 
density is expected to be self-averaging, i.e. that (6) and (7) agree in the thermodynamic limit $N \rightarrow \infty$.

The distribution of connectivities is taken to be maximally random compatible with the distribution of coordinations. Vertices $i \in \mathcal{N}_{\mu}$ and $j \in \mathcal{N}_{\nu}$ are connected with a probability proportional to $k_{i}^{\mu} k_{j}^{\nu}$. This can be expressed in terms of a fundamental distribution $p_{0}^{\mu \nu}$ of connectivities (between sites $i \in \mathcal{N}_{\mu}$ and $j \in \mathcal{N}_{\nu}$ )

as

$$
p_{0}^{\mu \nu}\left(c_{i j}\right)=\left(1-\frac{c^{\mu \nu}}{N_{v}}\right) \delta_{c_{i j}, 0}+\frac{c^{\mu \nu}}{N_{v}} \delta_{c_{i j}, 1}
$$

$$
\begin{aligned}
P\left(\boldsymbol{c} \mid\left\{\boldsymbol{k}_{i}\right\}\right)= & \left.\frac{1}{\mathcal{N}} \prod_{\mu}\left\{\prod_{i<j \in \mathcal{N}_{\mu}} p_{0}^{\mu \mu}\left(c_{i j}\right) \delta_{c_{i j}, c_{j i}}\right\} \times \prod_{\nu(>\mu)}\left\{\prod_{i \in \mathcal{N}_{\mu}} \prod_{j \in \mathcal{N}_{v}} p_{0}^{\mu \nu}\left(c_{i j}\right) \delta_{c_{i j}, c_{j i}}\right\}\right\} \\
& \times \prod_{\mu} \prod_{i \in \mathcal{N}_{\mu}}\left\{\prod_{\nu} \delta_{\Sigma_{j \in \mathcal{N}_{\nu}} c_{i j}, k_{i}^{v}}\right\}
\end{aligned}
$$

where $\mathcal{N}$ is a normalization constant, and the Kronecker-deltas enforce the prescribed coordinations. This is what is commonly referred to as a micro-canonical ensemble of connectivites. Alternatively one could generate a canonical ensemble by choosing $p\left(c_{i j}\right)=\left(1-k_{i}^{\mu} k_{j}^{\nu} /\left(c^{\mu \nu} N_{\nu}\right)\right) \delta_{c_{i j}, 0}+k_{i}^{\mu} k_{j}^{\nu} /\left(c^{\mu \nu} N_{v}\right) \delta_{c_{i j}, 1}$, independently for pairs of indices $i \in \mathcal{N}_{\mu}$ and $j \in \mathcal{N}_{\nu}$, which will reproduce only distributions of coordinations in the large $N$ limit. In this paper we use the micro-canonical approach for averaging.

The average of the logarithm in (7) is evaluated using the replica

$$
\overline{\rho(\lambda)}=\lim _{\varepsilon \searrow 0} \frac{-2}{N \pi} \operatorname{Im} \frac{\partial}{\partial \lambda} \lim _{n \rightarrow 0} \frac{1}{n} \ln \left\langle Z_{N}^{n}\right\rangle,
$$

with

$$
Z_{N}^{n}=\int \prod_{i a} \frac{\mathrm{d} u_{i a}}{\sqrt{2 \pi / \mathrm{i}}} \exp \left\{-\frac{\mathrm{i}}{2} \sum_{a} \sum_{i j} u_{i a}\left[\lambda_{\varepsilon} \delta_{i j}-M_{i j}\right] u_{j a}\right\} .
$$

Here $a=1, \ldots, n$ enumerates the replica. We initially describe the process for matrices of type (4), and briefly mention the modifications to treat matrices of type (5) with zero row-sum constraints later.

\subsection{Disorder average}

To evaluate the average, one uses integral representations of the Kronecker-deltas

$$
\delta_{\Sigma_{j \in \mathcal{N}_{v}} c_{i j}, k_{i}^{v}}=\int \frac{\mathrm{d} \varphi_{i}^{v}}{2 \pi} \mathrm{e}^{\mathrm{i} \varphi_{i}^{v}\left(\Sigma_{j \in \mathcal{N}_{v}} c_{i j}-k_{i}^{v}\right)} .
$$

The average of the replicated partition function for matrices of type (4) becomes

$$
\begin{aligned}
\left\langle Z_{N}^{n}\right\rangle= & \frac{1}{\mathcal{N}} \int \prod_{i \nu} \frac{\mathrm{d} \varphi_{i}^{\nu}}{2 \pi} \prod_{i a} \frac{\mathrm{d} u_{i a}}{\sqrt{2 \pi / \mathrm{i}}} \exp \left\{-\frac{\mathrm{i}}{2} \lambda_{\varepsilon} \sum_{i a} u_{i a}^{2}-\mathrm{i} \sum_{\mu \nu} \sum_{i \in N_{\mu}} \varphi_{i}^{v} k_{i}^{v}\right. \\
& +\sum_{\mu} \frac{c^{\mu \mu}}{2 N_{\mu}} \sum_{i \neq j \in \mathcal{N}_{\mu}}\left(\left\langle\exp \left\{\mathrm{i} K \sum_{a} u_{i a} u_{j a}\right\}\right\rangle_{\mu \mu} \mathrm{e}^{\mathrm{i} \varphi_{i}^{\mu}+\mathrm{i} \varphi_{j}^{\mu}}-1\right) \\
& \left.+\sum_{\mu<\nu} \frac{c^{\mu \nu}}{N_{\nu}} \sum_{i \in \mathcal{N}_{\mu}} \sum_{j \in \mathcal{N}_{\nu}}\left(\left\langle\exp \left\{\mathrm{i} K \sum_{a} u_{i a} u_{j a}\right\}\right\rangle_{\mu \nu} \mathrm{e}^{\mathrm{i} \varphi_{i}^{\nu}+\mathrm{i} \varphi_{j}^{\mu}}-1\right)\right\}
\end{aligned}
$$


where $\langle\ldots\rangle_{\mu \nu}$ represents an average over the $K_{i j}$ distribution, connecting vertices $i \in \mathcal{N}_{\mu}$ and $j \in \mathcal{N}_{\nu}$, which is as yet left open.

Decoupling of sites is achieved by introduction of the replicated 'densities'

$$
\rho^{(\mu \nu)}(\boldsymbol{u}, \varphi)=\frac{1}{N_{\mu}} \sum_{i \in \mathcal{N}_{\mu}} \prod_{a} \delta\left(u_{a}-u_{i a}\right) \delta\left(\varphi-\varphi_{i}^{\nu}\right)
$$

and their $\varphi$ integrated versions

$$
\rho^{(\mu \nu)}(\boldsymbol{u})=\int \mathrm{d} \varphi \rho^{(\mu \nu)}(\boldsymbol{u}, \varphi) \mathrm{e}^{\mathrm{i} \varphi} .
$$

It turns out that only the latter and their conjugate densities $\hat{\rho}^{(\mu \nu)}$ are needed, and equation (13) can be expressed as a functional integral

$$
\left\langle Z_{N}^{n}\right\rangle=\frac{1}{\mathcal{N}} \int \prod_{\mu \nu}\left\{\mathcal{D} \rho^{(\mu \nu)} \mathcal{D} \hat{\rho}^{(\mu \nu)}\right\} \exp \left\{N\left[G_{1}+G_{2}+G_{3}\right]\right\}
$$

with

$$
\begin{aligned}
G_{1} & =\frac{1}{2} \sum_{\mu \nu} f_{\mu} c^{\mu \nu}\left(\int \mathrm{d} \rho^{(\mu \nu)}(\boldsymbol{u}) \mathrm{d} \rho^{(\nu \mu)}(\boldsymbol{v})\left\langle\exp \left\{\mathrm{i} K \sum_{a} u_{a} v_{a}\right\}\right\rangle_{\mu \nu}-1\right) \\
G_{2} & =-\mathrm{i} \sum_{\mu \nu} f_{\mu} \int \mathrm{d} \boldsymbol{u} \hat{\rho}^{(\mu \nu)}(\boldsymbol{u}) \rho^{(\mu \nu)}(\boldsymbol{u}) \\
G_{3} & =\sum_{\mu} f_{\mu}\left\langle\ln \int \prod_{a} \frac{\mathrm{d} u_{a}}{\sqrt{2 \pi / \mathrm{i}}} \prod_{\nu} \frac{\left(\mathrm{i} \hat{\rho}^{(\mu \nu)}(\boldsymbol{u})\right)^{k^{\nu}}}{k^{\nu} !} \exp \left\{-\mathrm{i} \frac{\lambda_{\varepsilon}}{2} \sum_{a} u_{a}^{2}\right\}\right\rangle_{\mu} .
\end{aligned}
$$

Here we have exploited the symmetry relation $f_{\mu} c^{\mu \nu}=f_{\nu} c^{\nu \mu}$, introduced the shorthand notations $\mathrm{d} \rho^{(\mu \nu)}(\boldsymbol{u}) \equiv \mathrm{d} \boldsymbol{u} \rho^{(\mu \nu)}(\boldsymbol{u})$ for integrals over densities where appropriate, and $\langle\ldots\rangle_{\mu}$ in (19) for the average over the distribution of coordinations of sites in module $\mathcal{N}_{\mu}$.

\subsection{Replica symmetry and self-consistency equations}

The functional integral (16) is evaluated by the saddle point method. As in the extensively cross-connected case, the saddle point for this problem is expected to be both replica-symmetric and rotationally symmetric in the replica space. In the present context this translates to an ansatz of the form

$$
\begin{aligned}
& \rho^{(\mu \nu)}(\boldsymbol{u})=\rho_{0}^{(\mu \nu)} \int \mathrm{d} \pi^{(\mu \nu)}(\omega) \prod_{a} \frac{\exp \left[-\frac{\omega}{2} u_{a}^{2}\right]}{Z(\omega)}, \\
& \hat{\rho}^{(\mu \nu)}(\boldsymbol{u})=\hat{\rho}_{0}^{(\mu \nu)} \int \mathrm{d} \hat{\pi}^{(\mu \nu)}(\hat{\omega}) \prod_{a} \frac{\exp \left[-\frac{\hat{\omega}}{2} u_{a}^{2}\right]}{Z(\hat{\omega})},
\end{aligned}
$$

with normalization constants

$$
Z(\omega)=\int \mathrm{d} u \exp \left[-\frac{\omega}{2} u^{2}\right]=\sqrt{2 \pi / \omega},
$$

i.e. an uncountably infinite superposition of complex Gaussians (with $\operatorname{Re}[\omega] \geqslant 0$ and $\operatorname{Re}[\hat{\omega}] \geqslant 0)$ for the replicated densities and their conjugates $[12,22]$. The $\rho_{0}^{(\mu \nu)}, \hat{\rho}_{0}^{(\mu \nu)}$ in the expressions for $\rho^{(\mu \nu)}$ and $\hat{\rho}^{(\mu \nu)}$ in (20) are determined such that the densities $\pi^{(\mu \nu)}$ and $\hat{\pi}^{(\mu \nu)}$ are normalized. 
This ansatz translates path integrals over the replicated densities $\rho^{(\mu \nu)}$ and $\hat{\rho}^{(\mu \nu)}$ into path integrals over the densities $\pi^{(\mu \nu)}$ and $\hat{\pi}^{(\mu \nu)}$, and integrals over the normalization factors $\rho_{0}^{(\mu \nu)}$ and $\hat{\rho}_{0}^{(\mu \nu)}$. These integrals are evaluated using the saddle-pint method, giving rise to a pair of self-consistency equations for the densities $\pi^{(\mu \nu)}$ and $\hat{\pi}^{(\mu \nu)}$. Details are described in the appendix. In this case we get equations which closely resemble those derived in [12, 22], namely

$$
\begin{aligned}
& \hat{\pi}^{(\mu \nu)}(\hat{\omega})=\int \mathrm{d} \pi^{(\nu \mu)}\left(\omega^{\prime}\right)\left\langle\delta\left(\hat{\omega}-\hat{\Omega}\left(\omega^{\prime}, K\right)\right\rangle_{\mu \nu}\right. \\
& \pi^{(\mu \nu)}(\omega)=\left\langle\frac{k^{\nu}}{c^{\mu \nu}} \int\left\{\mathrm{d} \hat{\pi}^{(\mu)}\right\}_{k \backslash k^{\nu}} \delta\left(\omega-\Omega_{k \backslash k^{v}}^{(\mu)}\right)\right\rangle_{\mu}
\end{aligned}
$$

with

$$
\hat{\Omega}\left(\omega^{\prime}, K\right)=\frac{K^{2}}{\omega^{\prime}}, \quad \Omega_{k \backslash k^{\nu}}^{(\mu)}=\mathrm{i} \lambda_{\varepsilon}+\Sigma_{k \backslash k^{\nu}} \hat{\omega} .
$$

Here, we the use the shorthand notation $\left\{\mathrm{d} \hat{\pi}^{(\mu)}\right\}_{k \backslash k^{v}}$ to denote a product of integration measures of the form $\left\{\mathrm{d} \hat{\pi}^{(\mu)}\right\}_{k \backslash k^{v}} \equiv \prod_{\tilde{\nu}(\neq \nu)} \prod_{\ell_{\tilde{v}}=1}^{k^{\tilde{v}}} \mathrm{~d} \hat{\pi}^{(\mu \tilde{\nu})}\left(\hat{\omega}_{\ell_{\tilde{\nu}}}\right) \times \prod_{\ell_{v}=1}^{k^{\nu}-1} \mathrm{~d} \hat{\pi}^{(\mu \nu)}\left(\hat{\omega}_{\ell_{v}}\right)$ from which $\mathrm{d} \hat{\pi}^{(\mu \nu)}\left(\hat{\omega}_{k^{v}}\right)$ is excluded. An analogous construction applies to the sum $\Sigma_{k \backslash k^{v}} \hat{\omega}$ (see the appendix).

The structure of these equations suggests solving them via a population-based algorithm.

The spectral density is obtained from (6); note that only the explicit $\lambda$ dependence in $G_{3}$ in (19) or its replica-symmetric version (A.4) contributes. We obtain a formal result analogous to that obtained earlier for homogeneous systems, or for cross-connected modules of equal size with Poisson distributions of inter-module coordinations [22]

$$
\rho(\lambda)=\frac{1}{\pi} \operatorname{Re} \lim _{\varepsilon \searrow 0}\left[\sum_{\mu} f_{\mu} q_{d}^{(\mu)}\right]
$$

where

$$
\begin{aligned}
q_{d}^{(\mu)} & =\left\langle\int\left\{\mathrm{d} \hat{\pi}^{(\mu)}\right\}_{k}\left\langle u^{2}\right\rangle_{\{\hat{\omega}\}_{k}}\right\rangle_{\mu} \\
& =\left\langle\int\left\{\mathrm{d} \hat{\pi}^{(\mu)}\right\}_{k} \frac{1}{i \lambda_{\varepsilon}+\Sigma_{k} \hat{\omega}}\right\rangle_{\mu} .
\end{aligned}
$$

Here $\langle\ldots\rangle_{\{\hat{\omega}\}_{k}}$ is an average w.r.t. the Gaussian weight in terms of which $Z_{\lambda}\left(\Sigma_{k} \hat{\omega}\right)$ is defined.

It is important to note that the $\varepsilon \searrow 0$ limit of the (real part of the) integrand in (26) is singular in $\lambda$ for $\hat{\omega}$ combinations for which $\Sigma_{k} \hat{\omega}$ is purely imaginary. Indeed, writing $\Sigma_{k} \hat{\omega}=R_{k}+\mathrm{i} I_{k}$ with real $R_{k}$ and $I_{k}$, we have

$$
\operatorname{Re} \frac{1}{\mathrm{i} \lambda_{\varepsilon}+\Sigma_{k} \hat{\omega}}=\frac{\varepsilon+R_{k}}{\left(\varepsilon+R_{k}\right)^{2}+\left(\lambda+I_{k}\right)^{2}},
$$

so that for $R_{k}=0$ we have the limit

$$
\lim _{\varepsilon \searrow 0} \operatorname{Re} \frac{1}{i \lambda_{\varepsilon}+\Sigma_{k} \hat{\omega}}=\pi \delta\left(\lambda+I_{k}\right) .
$$

As demonstrated in [12], the contributions to (26) with $R_{k}=0$ can be identified with purepoint contributions to the spectral density, associated with localized states. In an evaluation of (25) and (26) via sampling from a population, one would miss these contributions to the 
spectral density, as finding a $\hat{\omega}$-combination for which $\lambda+I_{k}=0$-for $R_{k}=0$ they are according to the above analysis the only ones contributing in the $\varepsilon \searrow 0$-limit—would be a probability-zero event. Thus, in order to see the pure-point contributions, a small non-zero regularizing $\varepsilon$ must be kept, which amounts to replacing $\delta$-functions by Lorentzians of width $\varepsilon$, resulting in a density of states which is smoothed at the scale $\varepsilon$. A simultaneous evaluation of (26) for non-zero $\varepsilon$ and in the $\varepsilon \searrow 0$-limit then allows us to disentangle pure-point and continuous contributions to the total density of states (25). For further details on this matter we refer to [12].

If we are interested in spectra of the generalized graph Laplacians $L$ defined by (5) instead of the weighted adjacency matrices $M$, we need to evaluate

$$
\begin{aligned}
\left\langle Z_{N}^{n}\right\rangle=\frac{1}{\mathcal{N}} \int & \prod_{i \nu} \frac{\mathrm{d} \varphi_{i}^{v}}{2 \pi} \prod_{i a} \frac{\mathrm{d} u_{i a}}{\sqrt{2 \pi / \mathrm{i}}} \exp \left\{-\frac{\mathrm{i}}{2} \lambda_{\varepsilon} \sum_{i a} u_{i a}^{2}-\mathrm{i} \sum_{\mu \nu} \sum_{i \in N_{\mu}} \varphi_{i}^{\nu} k_{i}^{\nu}\right. \\
& +\sum_{\mu} \frac{c^{\mu \mu}}{2 N_{\mu}} \sum_{i \neq j \in \mathcal{N}_{\mu}}\left(\left\langle\exp \left\{-\mathrm{i} \frac{K}{2} \sum_{a}\left(u_{i a}-u_{j a}\right)^{2}\right\}\right\rangle_{\mu \mu} \mathrm{e}^{\mathrm{i} \varphi_{i}^{\mu}+\mathrm{i} \varphi_{j}^{\mu}}-1\right) \\
& \left.+\sum_{\mu<\nu} \frac{c^{\mu \nu}}{N_{\nu}} \sum_{i \in \mathcal{N}_{\mu}} \sum_{j \in \mathcal{N}_{v}}\left(\left\langle\exp \left\{-\mathrm{i} \frac{K}{2} \sum_{a}\left(u_{i a}-u_{j a}\right)^{2}\right\}\right\rangle_{\mu \nu} \mathrm{e}^{\mathrm{i} \varphi_{i}^{\nu}+\mathrm{i} \varphi_{j}^{\mu}}-1\right)\right\}
\end{aligned}
$$

instead of (13), the only difference being the translationally invariant form of the interactions in this case ${ }^{3}$. The structure of the theory developed above and the fixed point equations (22) and (23) remain formally unaltered, apart from a modification of the definition of $Z_{2}\left(\omega, \omega^{\prime}, K\right)$ of (A.6) due to the modified interaction term

$$
\begin{aligned}
Z_{2}\left(\omega, \omega^{\prime}, K\right) & =\int \mathrm{d} u \mathrm{~d} v \exp \left[-\frac{1}{2}\left(\omega u^{2}+\omega^{\prime} v^{2}+\mathrm{i} K(u-v)^{2}\right)\right] \\
& =Z\left(\omega^{\prime}+\mathrm{i} K\right) Z\left(\omega+\frac{K \omega^{\prime}}{K-\mathrm{i} \omega^{\prime}}\right)
\end{aligned}
$$

here expressed in terms of the normalization constants $Z(\cdot)$ of (21). As already noted in [12] this only requires a modified definition of $\hat{\Omega}\left(\omega^{\prime}, K\right)$ in $(24)$, namely

$$
\hat{\Omega}\left(\omega^{\prime}, K\right)=\frac{K \omega^{\prime}}{K-\mathrm{i} \omega^{\prime}},
$$

but leaves the the self-consistency equations otherwise unchanged.

\subsection{Cavity equations for finite instances}

Rather than studying the ensemble in the thermodynamic limit, one can also look at large but finite single instances. The method of choice to study these is the cavity approach [13], for which the additional structure coming from modularity does not cause any additional complication at all, and the original setup [13] applies without modification, apart from that related to generating large modular graphs with the prescribed statistics of inter- and intramodule connectivities.

Equations (11) of [13], when written in terms of the notation and conventions used in this paper, translate into

$$
\hat{\omega}_{\ell}^{(j)}=\frac{K_{j \ell}^{2}}{\omega_{\ell}^{(j)}}, \quad \omega_{j}^{(i)}=\mathrm{i} \lambda_{\varepsilon}+\sum_{\ell \in \partial j \backslash i} \hat{\omega}_{\ell}^{(j)},
$$

3 There is a typo, a missing minus sign, in front of the translationally invariant interaction term in the last unnumbered equation on $p 15$ of [12]. 
in which $\partial j$ denotes the set of vertices connected to $j$, and $\partial j \backslash i$ the set of neighbours of $j$, excluding $i$.

These equations can be solved iteratively [13] even for very large system sizes, showing fast convergence except at mobility edges, where we observe critical slowing-down.

The density of states for a single instance of a matrix $M$ is obtained from the self-consistent solution via

$$
\rho_{M}(\lambda)=\lim _{\varepsilon \searrow 0} \frac{1}{N \pi} \sum_{j} \operatorname{Re}\left[\frac{1}{\omega_{j}}\right]
$$

with

$$
\omega_{j}=\mathrm{i} \lambda_{\varepsilon}+\sum_{\ell \in \partial j} \hat{\omega}_{\ell}^{(j)}
$$

The modifications required to treat generalized graph Laplacians are once more straightforward.

To discuss the time complexity of the cavity algorithm for finite instances, note that the number of $\hat{\omega}_{\ell}^{(j)}$ variables is $\mathcal{O}(c N)$ for a system of $N$ vertices with average connectivity $c$. According to equation (30) each individual update requires one division and on average $c-1$ summations. As the number of update sweeps through, the system required for convergence is itself independent of system size (except at mobility edges); the computational effort to determine the spectral density for a single large instance at fixed resolution of $\lambda$ values scales linearly with $N$.

\section{Small-world networks}

Small-world networks can be constructed from any graph, by introducing a second, random connectivity component which introduces shortcuts in the original graph, as long as the second component is sufficiently weakly correlated with the first.

The standard example is a closed ring, with additional links between randomly chosen pairs along the ring. Alternatively, one could start with a regular random graph of fixed coordination $k_{i}=2$ (this gives an ensemble of loops with typical lengths diverging in the thermodynamic limit $N \rightarrow \infty$ ), and then introducing a second sparse connectivity component linking randomly chosen vertices of the original graph.

Clearly the original graph need not be a ring; it could be a $d$ dimensional lattice, a Bethe lattice, or a (regular) random graph of (average) connectivity different from 2 , and one could introduce several additional random connectivity components to create shortcuts.

In what follows, we look at (finitely coordinated) random graphs with several connectivity components between the vertices of the graphs. The setup is rather close to that of multimodular systems as described above, except that there is only a single module, having $m$ connectivity components linking the vertices of this single module.

The formal structure of the theory is therefore very similar to that described earlier and we just quote the final fixed point equations, and the result for the spectral density, without derivations.

We need to solve the following set of fixed point equations:

$$
\begin{aligned}
& \hat{\pi}^{(v)}(\hat{\omega})=\int \mathrm{d} \pi^{(\nu)}\left(\omega^{\prime}\right)\left\langle\delta\left(\hat{\omega}-\hat{\Omega}\left(\omega^{\prime}, K\right)\right\rangle_{v}\right. \\
& \pi^{(\nu)}(\omega)=\left\langle\frac{k^{\nu}}{c^{\nu}} \int\{\mathrm{d} \hat{\pi}\}_{k \backslash k^{v}} \delta\left(\omega-\Omega_{k \backslash k^{\nu}}\right)\right\rangle
\end{aligned}
$$


with

$$
\hat{\Omega}\left(\omega^{\prime}, K\right)=\frac{K^{2}}{\omega^{\prime}}, \quad \Omega_{k \backslash k^{v}}=\mathrm{i} \lambda_{\varepsilon}+\Sigma_{k \backslash k^{\nu}} \hat{\omega},
$$

where now $\langle\ldots\rangle_{v}$ in (33) denotes an average over the weight distribution of the $v$ th coupling component $\left\{K_{i j}^{(v)}\right\}$, and the average $\langle\ldots\rangle$ in (34) is over the distribution of $m$-dimensional coordinations $\boldsymbol{k}_{i}=\left(k_{i}^{v}\right)$ with $\left\langle k_{i}^{v}\right\rangle=c^{v}$. The (average) spectral density is then given by

$$
\rho(\lambda)=\frac{1}{\pi} \operatorname{Re} \lim _{\varepsilon \searrow 0}\left\langle\int\{\mathrm{d} \hat{\pi}\}_{k} \frac{1}{\mathrm{i} \lambda_{\varepsilon}+\Sigma_{k} \hat{\omega}}\right\rangle .
$$

If one were to look at the graph Laplacian for this type of small-world network, rather than at weighted adjacency matrices, one would once more only have to substitute (29) for $\hat{\Omega}$ in (35), as discussed in for the multi-modular case above.

\section{Results}

\subsection{Modular systems}

For the multi-modular systems, there are clearly far too many possible parameters and parameter combinations to even begin to attempt giving an overview of the phenomena one might see in such systems. Hence, we restrict ourselves to just one illustrative example chosen to highlight how the total density of states in different parts of the spectrum may be dominated by contributions of local densities of states of specific sub-modules.

We present a system consisting of three modules, with fractions $f_{1}=1 / 2, f_{2}=1 / 3$, and $f_{3}=1 / 6$ of the system, respectively. Modules 1 and 3 have fixed intra-modular connectivities with coordinations 3 and 2, respectively, while module 2 has Poisson connectivity with average 2. Inter-module connectivities are all Poissonian with averages $c^{12}=1$ and $c^{13}=c^{23}=1 / 2$ $\left(c^{21}, c^{31}\right.$ and $c^{32}$ follow from the consistency requirements). Non-zero couplings are chosen bi-modal $\pm 1 / \sqrt{c}_{t}$ with $c_{t}=\sum_{\mu \nu} f_{\mu} c^{\mu \nu}$ apart from intra-module couplings in modules 1 and 3 , which have values of $\pm 1 / 2 \sqrt{c}_{t}$ and $\pm 2 / \sqrt{c}_{t}$, respectively.

Figure 1 shows the results for this system. We observe that the central cusp at $\lambda=0$ and the $\delta$-function contributions to the total density of states at $\lambda=0$ and at $\lambda \simeq \pm 0.463$ essentially originate from module 2 (with Poisson connectivity of average coordination 2); a regularizing $\varepsilon=10^{-4}$ has been used to exhibit the $\delta$-function contributions. The humps at the edges of the spectrum mainly come from module 3 with the fixed coordination 2 , whereas the shape of the shoulders at intermediate $\lambda$ values are mostly determined by the largest module 1 with fixed coordination 3 . Note that there are small tails of localized states for $|\lambda| \gtrsim 2.415$.

We found results computed for a single instance of this modular structure containing $N=60000$ vertices to be virtually indistinguishable from the ensemble results, except for finite sample fluctuations in the extreme tails where the expected DOS becomes too small to expect more than a few eigenvalues for the $N=60000$ system.

The $\delta$-peaks at $\lambda \simeq \pm 0.463$ originate from isolated dimers as part of module 2 which remain isolated upon cross-linking the different modules. For the three-module system in question, the weight $a_{2}$ of each of the $\delta$-peaks can be shown to be $\frac{1}{6} \mathrm{e}^{-8} \simeq 5.6 \times 10^{-5}$ in the thermodynamic limit, compatible with a rough estimate of $a_{2}=(5 \pm 1) \times 10^{-5}$ from our numerical ensemble results. We note in passing that pure-point contributions to the spectral density would be generated by many other finite isolated clusters; the ones with the next highest weight would be generated by isolated open trimers, but these are more than an order 


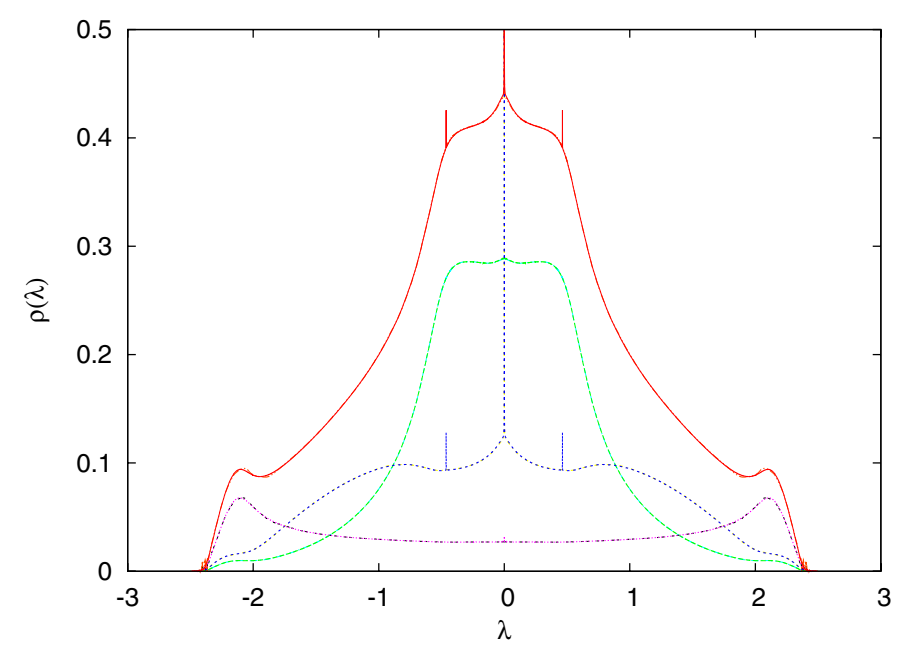

Figure 1. Spectrum of the three-module system described in the main text (red full line), along with its unfolding according to contributions of local densities of states contributed by the three sub-modules as suggested by equation (39) (green long dashed: module 1, blue short dashed: module 2, and magenta dot-dashed: module 3). Ensemble results are displayed together with the corresponding results for a single instance of size $N=60000$. The former are plotted on top of the latter, so colour-coded lines for the finite instance results (light blue: module 1, yellow: module 2, black dashed: module 3, and light-red dashed for the total DOS) remain basically invisible due to the nearly perfect match.

of magnitude less likely to occur, so that we have not picked them up at the precision with which we have performed the $\lambda$ scan in figure 1 .

\subsection{Small-world networks}

The small-world networks we consider here have a small fraction of long-range connections added to a regular random graph of fixed coordination 2. The system without long-range interactions is effectively an infinite ring; it can be diagonalized analytically; for couplings of unit strength it has a band of extended states for $|\lambda| \leqslant 2$, and the density of states exhibits the typical integrable van Hove singularity $\rho(\lambda) \sim|| \lambda|-2|^{-1 / 2}$ of a one-dimensional regular system.

When a small amount of weak long-range interactions is introduced into the system, this central band will initially slightly broaden, and the van Hove singularity gets rounded (the integrable divergence disappears). As the strength of the long-range connections is increased, the central band is widened further. At the same time the density of states acquires some structure, which becomes more intricate, as the strength of the long-range interactions is increased, including side-peaks which themselves acquire sub-structure, and typically a depression of the DOS near the location of the original band edge. This depression deepens with increasing strength of the long-range interactions, and eventually becomes a proper band gap, which we find to be populated only by localized states. Further increase in interaction strength introduces ever more structure, including depressions in the DOS inside side-bands which in turn develop into proper band gaps.

Figure 2 shows a system for which the average additional long-range coordination is $c=0.5$, so that long-range interactions are associated with fewer than half of the nodes on the 


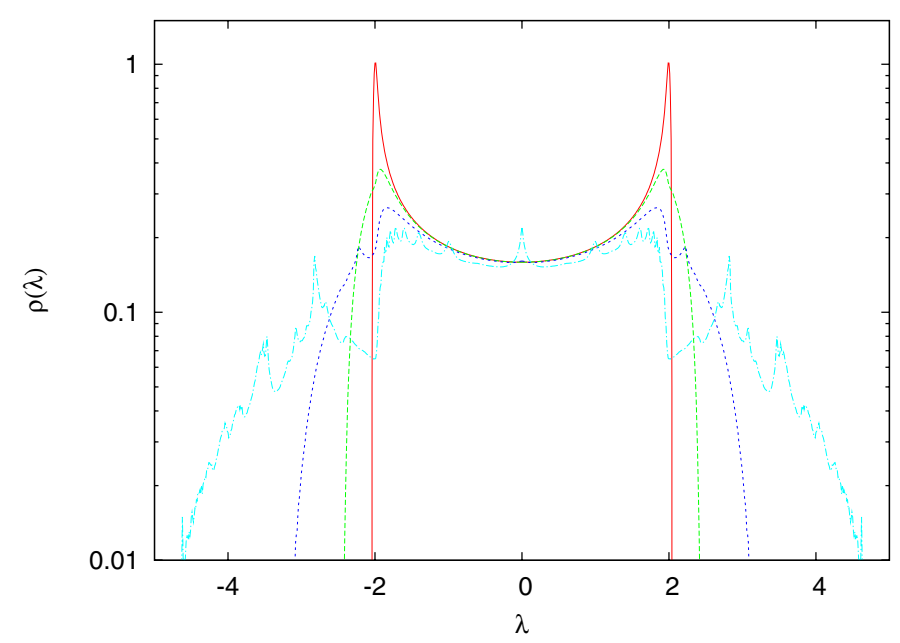

Figure 2. Central part of the spectrum the small-world system described in the text. The four curves correspond to long-range interactions of strengths $J=0.1$ (red full line), $J=0.5$ (green long dashed), $J=1.0$ (blue short dashed), and $J=2.0$ (light-blue dot-dashed).

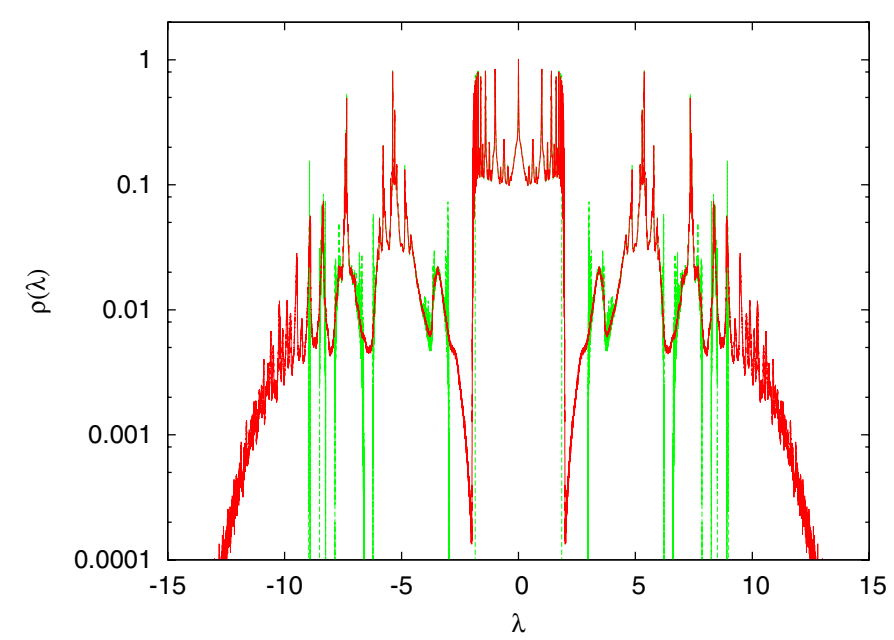

Figure 3. Spectrum of the small-world system with $c=0.5$ at $J=5$, showing both the DOS of the extended states (green long dashed line) and the total DOS including contributions from localized states (red full line). A regularizing $\varepsilon=10^{-3}$ has been used to exhibit the latter.

ring. The figure displays the central region of the spectrum for a range of interaction strengths of the long-range couplings, which exhibit increasing amounts of structure with increasing interaction strengths.

Figure 3 shows the entire spectrum of this system at $J=5$, and separately exhibits the contribution of the continuous spectrum. Now four distinct separate side-bands of continuous states can clearly be distinguished on each side of the central band, with proper band gaps (filled with localized states) between them. 
The spectrum shown in figure 3 displays structure at many levels. To mention just two of the more prominent ones: the original edge of the central band develops a sequence of peaks which extends into the localized region. Side-bands too acquire multi-peak structures, with individual peaks exhibiting further sub-structure.

Subject to limitations of computational power, our algorithm is able to exhibit these structures to any desired level of accuracy, though in some regions-predominantly at band edges - our data for the continuous DOS remain somewhat noisy; we suspect that in such regions there is a set of localized states that become dense in the thermodynamic limit, which is responsible for this phenomenon. Also we have a localization transition at every band edge which may well induce critical slowing down in the population dynamics algorithm by which we obtain spectral densities. Quite possibly because of this, finite population-size effects in the population dynamics are much stronger in the present small-world system than in the simpler systems without side-bands studied before [12, 13, 22].

We have attempted to verify the localization transitions using numerical diagonalization and computations of inverse participation ratios [26, 27] in finite instances of increasing size, but the convergence to asymptotic trends is extremely slow. Although we have gone to system sizes as large as $N=3200$ for this system, the numerical results, while compatible with those derived from our population dynamics algorithm, are still not forceful enough to strongly support them. These aspects clearly deserve further study. In this respect a recent result of Metz et al [28], who managed to compute IPRs within a population dynamics approach, could well provide the method of choice to clarify the situation, though we have not yet implemented their algorithm.

\subsection{Graph Laplacians}

From a dynamical point of view, graph Laplacians (5) are in many ways more interesting than the corresponding connectivity matrices (4), as they could be used to analyse e.g. diffusive transport on graphs, to give vibrational modes of structures described by these graphs, or to define the kinetic energy component of random Schrödinger operators. We have accordingly also looked at spectra of the graph Laplacian corresponding to the small-world-type structures discussed in the previous section.

For the regular random graph of fixed coordination 2, the spectrum of the graph Laplacian is just a shifted version of the spectrum of the connectivity matrix. As for the latter, by adding a small amount of weak long-range interactions, this translated band initially broadens slightly, and the van Hove singularities disappear. More or stronger long-range interactions do, however, not appear to create much structure in the initial band. The tails at the lower band edge do acquire structure and eventually develop proper band gaps, populated only by localized states, much as for the connectivity matrix.

Figure 4 shows the spectrum of the graph Laplacian for a small-world system with the same parameters as in figure 3. We recognize a main band of extended states for $-5.59 \lesssim \lambda \lesssim-0.037$ and four side bands, two of which are very narrow; they are centred around $\tilde{\lambda} \simeq-7.2$ and $\lambda \simeq-7.3$, and are barely distinguishable as separate bands on the scale of the figure. Although the data for $\lambda<-20$ appear noisy, the fine structure in this region of the spectrum is actually accurate; as shown in the inset, we found them to be very well reproduced by high precision exact diagonalization of an ensemble of $10^{4}$ matrices of size $1600 \times 1600$, using a fine binning (5000 bins across the entire spectrum, thus $\Delta \lambda \simeq 8 \times 10^{-3}$ ), to achieve sufficient resolution of details.

The appearance of several bands of extended states, separated by gaps which are populated only by localized states, implies that transport processes such as diffusion will exhibit several 


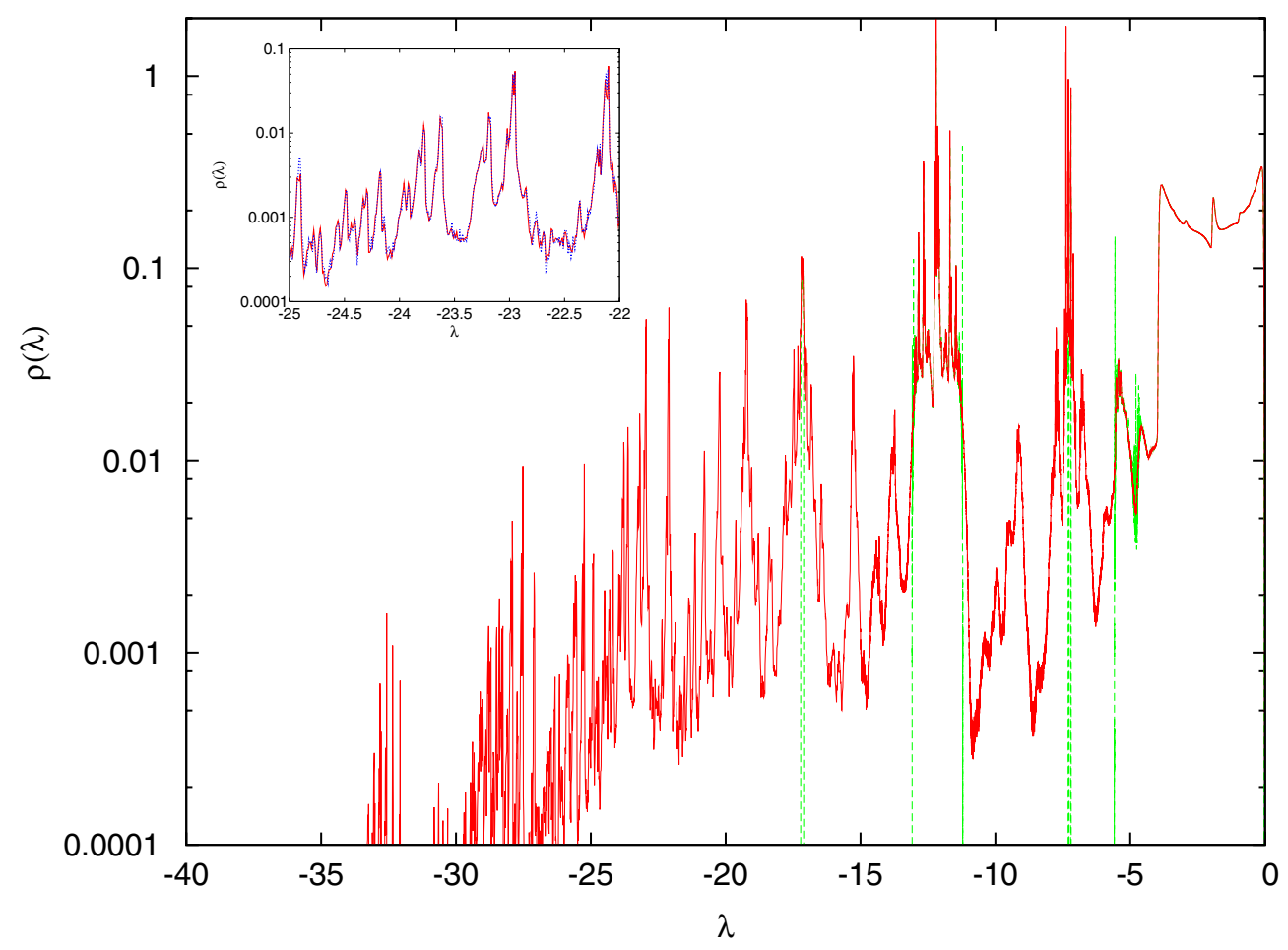

Figure 4. Spectrum of the graph Laplacian for a small-world system with $c=0.5$ at $J=5$, showing both the DOS of the extended states (green long dashed line) and the total DOS including contributions from localized states (red full line), regularized at $\varepsilon=10^{-3}$. The inset compares the population dynamics results for the total DOS (red full line) with results of direct numerical diagonalization (blue short dashed line) in the range $-25 \leqslant \lambda \leqslant-22$.

distinct time scales for such systems. Given the way in which the system is constructed, the appearance of two time scales would not be surprising, as diffusion takes place both along the ring and via short cuts. The fact that there are several such time scales would not seem obvious, though.

Another feature which becomes apparent only by zooming into the region of very small $|\lambda|$ is the appearance of a mobility edge at $\lambda_{c} \simeq-0.037$ and a region of localized states for $\lambda>\lambda_{c}$. The behaviour of the spectral density in the localized region $\lambda_{c} \leqslant \lambda \leqslant 0$ shows singular Lifshitz-type behaviour [29]. For systems with a range of different parameters, both for the average number $c$ of long-range connections per site, and for their strength $J$, we find it to be compatible with the functional form

$$
\rho(\lambda) \simeq a \exp \left(-b /|\lambda|^{2 / 3}\right)
$$

with $a$ and $b$ depending on $c$ and $J$. For the $c=0.5, J=5$ system shown in figure 5 we have $a=4.0 \pm 0.1$ and $b=0.49 \pm 0.003$. Three parameter fits which attempt to determine the $|\lambda|$ power in the exponential of (37) do give powers slightly different from 2/3 at comparable values of reduced $\chi^{2}$, but the uncertainties of individual parameters are much larger. It may be worth mentioning that we have observed similar Lifshitz tails also for Laplacians of simple Poisson random graphs, both below and above the percolation transition, and for Laplacians corresponding to modular random graphs such as the one studied in section 4.1. 


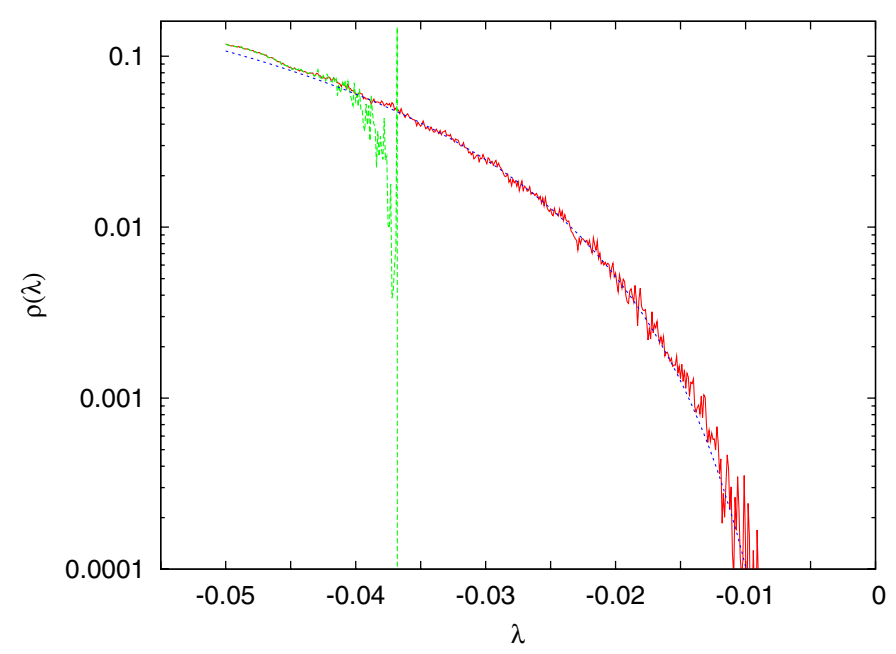

Figure 5. Lifshitz tail for small $|\lambda|$. Shown are the band edge of the extended states (green long dashed line), the total DOS including contributions from localized states (red full line), and (37) (blue short dashed), based on a fit of the data in the interval $-0.035 \leqslant \lambda \leqslant-0.0075$.

\section{Conclusions}

We have computed spectra of matrices describing random graphs with modular or small-world structure, looking both at connectivity matrices and at (weighted) graph Laplacians. Spectra are evaluated for random matrix ensembles in the thermodynamic limit using replica, and for large single instances using the cavity method. We find excellent agreement between the two sets of results if the single instances are sufficiently large; graphs containing $N=\mathcal{O}\left(10^{4}-10^{5}\right)$ vertices are typically required to achieve agreement with relative errors below $10^{-3}$. The ensemble and single instance results are in turn in excellent agreement with results of direct numerical diagonalizations, though averages over many samples are required for the latter due to the comparatively moderate sample sizes that can be handled in the direct diagonalization approach. As explained in section 2.4, we can associate pure-point contributions to the spectral density with singular contributions to the integrals (26) which can be exhibited by keeping small non-zero regularizing values of $\varepsilon$ while evaluating those integrals via sampling from a population.

For a multi-modular system we have seen, by way of example, how the total density of states in different parts of the spectrum may be dominated by contributions of local densities of states of specific sub-modules. The ability to identify such contributions may well become a useful diagnostic tool in situations where one needs to study the topology of modular systems for which plausible null models of their compositions are available.

For small-world systems, we have seen how the introduction of shortcuts in a regular graph adds structure to the spectrum of the original regular random graph from which the small-world system is derived. Depending on parameters, this may include the possibility of having one or several satellite bands of extended states separated from the original band by gaps that are populated only by localized states. Whenever this happens for (weighted) 
graph Laplacians this implies the introduction of different time scales for diffusive transport described by these Laplacians.

For graph Laplacians we typically observe a region of localized states at small $|\lambda|$ where the density of states exhibits singular Lifshitz-type behaviour. We note that the existence of a small $|\lambda|$ mobility edge implies that these systems will exhibit a finite maximum relaxation time for global diffusive modes though there is no corresponding upper limit for the relaxation times for local modes.

We iterate that our methods are completely general concerning the modular structure of the matrices. Concerning connectivity distributions, the only requirements are that they are maximally random subject only to the constraints coming from prescribed degree distributions. Modular graphs with additional topological constraints beyond degree distributions could be handled by suitably adapting the techniques of [23].

\section{Appendix. Replica-symmetric analysis}

The evaluation of the averaged replicated partition function (16) within the replica symmetric formulation when re-expressed in terms of path integrals over the densities $\pi^{(\mu \nu)}$ and $\hat{\pi}^{(\mu \nu)}$ and the normalization factors $\rho_{0}^{(\mu \nu)}$ and $\hat{\rho}_{0}^{(\mu \nu)}$ gives rise to

$$
\left\langle Z_{N}^{n}\right\rangle=\frac{1}{\mathcal{N}} \int \prod_{\mu \nu}\left\{\mathcal{D} \pi^{(\mu \nu)} \mathcal{D} \hat{\pi}^{(\mu \nu)} \mathrm{d} \hat{\rho}_{0}^{(\mu \nu)} \mathrm{d} \rho_{0}^{(\mu \nu)}\right\} \exp \left\{N\left[G_{1}+G_{2}+G_{3}\right]\right\},
$$

with

$$
\begin{aligned}
G_{1} \simeq \frac{1}{2} \sum_{\mu \nu} f_{\mu} c^{\mu \nu}\left[\left(\rho_{0}^{(\mu \nu)} \rho_{0}^{(\nu \mu)}-1\right)\right. & \\
& \left.+n \rho_{0}^{(\mu \nu)} \rho_{0}^{(\nu \mu)} \int \mathrm{d} \pi^{(\mu \nu)}(\omega) \mathrm{d} \pi^{(\nu \mu)}\left(\omega^{\prime}\right)\left\langle\ln \left[\frac{Z_{2}\left(\omega, \omega^{\prime}, K\right)}{Z(\omega) Z\left(\omega^{\prime}\right)}\right]\right\rangle_{\mu \nu}\right], \\
G_{2} \simeq & -\sum_{\mu \nu} f_{\mu} \mathrm{i} \hat{\rho}_{0}^{(\mu \nu)} \rho_{0}^{(\mu \nu)}\left[1+n \int \mathrm{d} \hat{\pi}^{(\mu \nu)}(\hat{\omega}) \mathrm{d} \pi^{(\mu \nu)}(\omega) \ln \left[\frac{Z(\hat{\omega}+\omega)}{Z(\hat{\omega}) Z(\omega)}\right]\right], \\
G_{3} \simeq & \sum_{\mu} f_{\mu}\left[\sum_{\nu}\left(\left\langle\ln \left(\frac{\left(\mathrm{i} \hat{\rho}_{0}^{(\mu \nu)}\right)^{k^{\nu}}}{k^{\nu !}}\right)\right\rangle_{\mu}\right)+n\left\langle\int\left\{\mathrm{d} \hat{\pi}^{(\mu)}\right\}_{k} \ln \left[\frac{Z_{\lambda}\left(\Sigma_{k} \hat{\omega}\right)}{\{Z\}_{k}}\right]\right\rangle_{\mu}\right] .
\end{aligned}
$$

Here, we have introduced shorthand notations for products of integration measures: $\left\{\mathrm{d} \hat{\pi}^{(\mu)}\right\}_{k} \equiv \prod_{\nu} \prod_{\ell_{v}=1}^{k^{v}} \mathrm{~d} \hat{\pi}^{(\mu \nu)}\left(\hat{\omega}_{\ell_{v}}\right)$, for products of partition functions: $\{Z\}_{k} \equiv$ $\prod_{v} \prod_{\ell_{v}=1}^{k^{v}} Z\left(\hat{\omega}_{\ell_{v}}\right)$, and for $\hat{\omega}$-sums: $\Sigma_{k} \hat{\omega} \equiv \sum_{v} \sum_{\ell_{v}=1}^{k^{v}} \hat{\omega}_{\ell_{v}}$. Furthermore, we have introduced the partition functions

$Z_{\lambda}\left(\Sigma_{k} \hat{\omega}\right)=\int \frac{\mathrm{d} u}{\sqrt{2 \pi / \mathrm{i}}} \exp \left[-\frac{1}{2}\left(\mathrm{i} \lambda_{\varepsilon}+\Sigma_{k} \hat{\omega}\right) u^{2}\right]=\left(\frac{\mathrm{i}}{i \lambda_{\varepsilon}+\Sigma_{k} \hat{\omega}}\right)^{1 / 2}$

and

$Z_{2}\left(\omega, \omega^{\prime}, K\right)=\int \mathrm{d} u \mathrm{~d} v \exp \left[-\frac{1}{2}\left(\omega u^{2}+\omega^{\prime} v^{2}-2 \mathrm{i} K u v\right)\right]=\frac{2 \pi}{\sqrt{\omega \omega^{\prime}+K^{2}}}$. 
The normalization constant $\mathcal{N}$ in (A.1) is given by

$$
\begin{aligned}
\mathcal{N}=\int \prod_{i \nu} \frac{\mathrm{d} \varphi_{i}^{\nu}}{2 \pi} & \exp \left\{\sum_{\mu} \frac{c^{\mu \mu}}{2 N_{\mu}} \sum_{i \neq j \in \mathcal{N}_{\mu}}\left(\mathrm{e}^{\mathrm{i} \varphi_{i}^{\mu}+\mathrm{i} \varphi_{j}^{\mu}}-1\right)\right. \\
& \left.+\sum_{\mu<\nu} \frac{c^{\mu \nu}}{N_{\nu}} \sum_{i \in \mathcal{N}_{\mu}} \sum_{j \in \mathcal{N}_{\nu}}\left(\mathrm{e}^{\mathrm{i} \varphi_{i}^{\nu}+\mathrm{i} \varphi_{j}^{\mu}}-1\right)-\mathrm{i} \sum_{\mu \nu} \sum_{i \in N_{\mu}} \varphi_{i}^{\nu} k_{i}^{\nu}\right\} .
\end{aligned}
$$

Site decoupling is achieved by introducing

$$
\rho_{0}^{(\mu \nu)}=\frac{1}{N_{\mu}} \sum_{i \in \mathcal{N}_{\mu}} \mathrm{e}^{\mathrm{i} \varphi_{i}^{v}}
$$

and a corresponding set of conjugate-order parameters to enforce these definitions. Note that we use a notation previously employed for normalization factors of replicated densities. This duplication is intentional, as it reveals terms in the numerator and denominator of (A.1) exhibiting the same exponential scaling in $N$ when evaluated at the saddle point, and hence cancel. We get

$$
\begin{aligned}
\mathcal{N}=\int \prod_{\mu \nu} \frac{\mathrm{d} \hat{\rho}_{0}^{(\mu \nu)} \mathrm{d} \rho_{0}^{(\mu \nu)}}{2 \pi / N_{\mu}} \exp \left\{N \left[\frac{1}{2} \sum_{\mu \nu} f_{\mu} c^{\mu \nu}\left(\rho_{0}^{(\mu \nu)} \rho_{0}^{(\nu \mu)}-1\right)\right.\right. \\
\left.\left.-\mathrm{i} \sum_{\mu \nu} f_{\mu} \hat{\rho}_{0}^{(\mu \nu)} \rho_{0}^{(\mu \nu)}+\sum_{\mu \nu} f_{\mu}\left(\left\langle k^{\nu} \ln \mathrm{i} \hat{\rho}_{0}^{(\mu \nu)}\right\rangle_{\mu}-\left\langle\ln k^{\nu} !\right\rangle_{\mu}\right)\right]\right\},
\end{aligned}
$$

which is also evaluated by the saddle point method.

Before we derive the saddle point conditions, we note that the functions $G_{1}, G_{2}$ and $G_{3}$ in the numerator of (A.1) contain both $\mathcal{O}(1)$ and $\mathcal{O}(n)$ contributions in the $n \rightarrow 0$ limit, such that the integrand in the numerator contains both terms that scale exponentially in $N$ and in $N n$. The denominator $(\mathcal{N})$, however, scales exponentially in $N$. Since the ratio (A.1) scales exponentially in $N n$, the $\mathcal{O}(1)$ contributions to $G_{1}, G_{2}$ and $G_{3}$ should cancel with those of $\mathcal{N}$ at the saddle point, which is indeed the case.

Evaluating first the stationarity conditions for $G=G_{1}+G_{2}+G_{3}$ at $\mathcal{O}(1)$ gives

$$
\mathrm{i} \hat{\rho}_{0}^{(\mu \nu)}=c^{\mu \nu} \rho_{0}^{(v \mu)} \quad \text { and } \quad \rho_{0}^{(\mu \nu)}=\frac{\left\langle k^{\nu}\right\rangle_{\mu}}{\mathrm{i} \hat{\rho}_{0}^{(\mu \nu)}}=\frac{c^{\mu \nu}}{\mathrm{i} \hat{\rho}_{0}^{(\mu \nu)}}
$$

from which we obtain

$$
\mathrm{i} \hat{\rho}_{0}^{(\mu \nu)} \rho_{0}^{(\mu \nu)}=c^{\mu \nu} \quad \text { and } \quad \rho_{0}^{(\mu \nu)} \rho_{0}^{(\nu \mu)}=1 .
$$

The stationarity conditions for the saddle point of $\mathcal{N}$ are exactly the same. Since (A.11) exhibits the gauge symmetry

$$
\rho_{0}^{(\mu \nu)} \rightarrow \rho_{0}^{(\mu \nu)} a^{(\mu \nu)}, \quad \mathrm{i} \hat{\rho}_{0}^{(\mu \nu)} \rightarrow \mathrm{i} \hat{\rho}_{0}^{(\mu \nu)} / a^{(\mu \nu)}, \quad \rho_{0}^{(\nu \mu)} \rightarrow \rho_{0}^{(\nu \mu)} / a^{(\mu \nu)},
$$

the correct scaling in $N n$ is obtained, provided that the same gauge is adopted in both the numerator and the denominator of (A.1). The saddle point contribution is determined from stationarity conditions with respect to variations of the $\pi^{(\mu \nu)}(\omega)$ and the $\hat{\pi}^{(\mu \nu)}(\hat{\omega})$.

Using (A.11), the stationarity conditions for the $\pi^{(\mu \nu)}(\omega)$ read

$$
\int \mathrm{d} \hat{\pi}^{(\mu v)}(\hat{\omega}) \ln \left[\frac{Z(\hat{\omega}+\omega)}{Z(\hat{\omega}) Z(\omega)}\right]=\int \mathrm{d} \pi^{(v \mu)}\left(\omega^{\prime}\right)\left\langle\ln \frac{Z_{2}\left(\omega, \omega^{\prime}, K\right)}{Z(\omega) Z\left(\omega^{\prime}\right)}\right\rangle_{\mu \nu}+\phi_{\mu \nu}
$$

with $\phi_{\mu \nu}$ a Lagrange multiplier to enforce the normalization of $\pi^{(\mu \nu)}$. 
The stationarity conditions for the $\hat{\pi}^{(\mu \nu)}(\hat{\omega})$ are

$$
c^{\mu \nu} \int \mathrm{d} \pi^{(\mu \nu)}(\omega) \ln \left[\frac{Z(\hat{\omega}+\omega)}{Z(\hat{\omega}) Z(\omega)}\right]=\left\langle k^{\nu} \int\left\{\mathrm{d} \hat{\pi}^{(\mu)}\right\}_{k \backslash k^{\nu}} \ln \frac{Z_{\lambda}\left(\hat{\omega}+\Sigma_{k \backslash k^{\nu}} \hat{\omega}\right)}{\{Z\}_{k \backslash k^{v}}}\right\rangle_{\mu}+\hat{\phi}_{\mu \nu}
$$

where $\hat{\phi}_{\mu \nu}$ is the Lagrange multiplier to enforce the normalization of $\hat{\pi}^{(\mu \nu)}(\hat{\omega})$, and where $\left\{\mathrm{d} \hat{\pi}^{(\mu)}\right\}_{k \backslash k^{\nu}}$ denotes the product $\left\{\mathrm{d} \hat{\pi}^{(\mu)}\right\}_{k}$ of integration measures from which $\mathrm{d} \hat{\pi}^{(\mu \nu)}\left(\hat{\omega}_{k^{\nu}}\right)$ is excluded, i.e. the product $\left\{\mathrm{d} \hat{\pi}^{(\mu)}\right\}_{k \backslash k^{v}} \equiv \prod_{\tilde{v}(\neq v)} \prod_{\ell_{\tilde{v}}=1}^{k^{\tilde{\nu}}} \mathrm{d} \hat{\pi}^{(\mu \tilde{v})}\left(\hat{\omega}_{\ell_{\tilde{\nu}}}\right) \times \prod_{\ell_{v}=1}^{k^{v}-1} \mathrm{~d} \hat{\pi}^{(\mu \nu)}\left(\hat{\omega}_{\ell_{v}}\right)$. Analogous constructions apply to the product $\{Z\}_{k \backslash k^{v}}$ and the sum $\Sigma_{k \backslash k^{v}} \hat{\omega}$.

Following [24, 25], the stationarity conditions for $\pi^{(\mu \nu)}(\omega)$ and $\hat{\pi}^{(\mu \nu)}(\hat{\omega})$ are rewritten in the form (22)-(24) given in section 2.3. These equations generalize those obtained in [12,22] for the unstructured and two-module cases, respectively.

\section{References}

[1] Newman M E J, Strogatz S H and Watts D J 2001 Random graphs with arbitrary degree distributions and their applications Phys. Rev. E 64026118

[2] Albert R and Barabási A-L 2002 Statistical mechanics of complex networks Rev. Mod. Phys. 74 47-97

[3] Newman M E J 2003 The structure and function of complex networks SIAM Rev. 45 167-256

[4] Dorogovtsev S N and Mendes J F F 2003 Evolution of Networks: From Biological Networks to the Internet and $W W W$ (Oxford: Oxford University Press)

[5] Dorogovtsev S N, Goltsev A V, Mendes J F F and Samukhin A N 2003 Spectra of complex networks Phys. Rev. E 68046109

[6] Cvetković D, Doob M and Sachs H 1995 Spectra of Graphs-Theory and Applications 3rd edn (Heidelberg: JA Barth)

[7] Bollobàs B 2001 Random Graphs (Cambridge: Cambridge University Press)

[8] Farkas I, Derény I, Barabási A L and Vicsek T 2001 Spectra of real world graphs: beyond the semi-circle law Phys. Rev. E 64026704

[9] Newman M E J 2006 Modularity and community structure in networks Proc. Natl Acad. Sci. USA 103 8577-82

[10] Rodgers G J and Bray A J 1988 Density of states of a sparse random matrix Phys. Rev. B 37 3557-62

[11] Bray A J and Rodgers G J 1988 Diffusion in a sparsely connected space: a model for glassy relaxation Phys. Rev. B 38 11461-70

[12] Kühn R 2008 Spectra of sparse random matrices J. Phys. A: Math. Theor. 41295002

[13] Rogers T, Pérez-Castillo I, Takeda K and Kühn R 2008 Cavity approach to the spectral density of sparse symmetric random matrices Phys. Rev. E 78031116

[14] Abou-Chacra R, Thouless D J and Anderson P W 1973 A self-consistent theory of localization J. Phys. C: Solid-State Phys. 6 1734-52

[15] Biroli G and Monasson R 1999 A single defect approximation for localized states on random lattices J. Phys. A: Math. Gen. 32 L255-61

[16] Semerjian G and Cugliandolo L F 2002 Sparse random matrices: the eigenvalue spectrum revisited J. Phys. A: Math. Gen. 35 4837-51

[17] Nagao T and Rodgers G J 2008 Spectral density of complex networks with a finite mean degree J. Phys. A: Math. Theor. 41265002

[18] Rodgers G J, Austin K, Kahng B and Kim D 2005 Eigenvalue spectra of complex networks J. Phys. A: Math. Gen. 38 9431-7

[19] Kim D and Kahng B 2007 Spectral densities of scale-free networks Chaos 17026115

[20] Goh K I, Kahng B and Kim D 2001 Spectra and eigenvectors of scale-free networks Phys. Rev. E 64051903

[21] Mitrović M and Tadić B 2009 Spectral and dynamical properties in classes of sparse networks with mesoscopic inhomogeneity Phys. Rev. E 80026123

[22] Ergün G and Kühn R 2009 Spectra of modular random graphs J. Phys. A: Math. Theor. 42395001

[23] Rogers T, Pérez Vicente C, Takeda K and Pérez-Castillo I 2010 Spectral density of random graphs with topological constraints J. Phys. A: Math. Theor. 43195002

[24] Monasson R 1998 Optimization problems and replica symmetry breaking in finite connectivity spin-glasses J. Phys. A: Math. Gen. 31 513-29

[25] Mézard M and Parisi G 2001 The bethe lattice spin glass revisited Eur. Phys. J. B 20 217-33 
[26] Evangelou S N and Economou E N 1992 Spectral density singularities, level statistics. and localization in a sparse random matrix ensemble Phys. Rev. Lett. 68 361-4

[27] Evangelou S N 1992 A numerical study of sparse random matrices J. Stat. Phys. 69 361-83

[28] Metz F, Neri I and Bollé D 2010 On the localization transition in symmetric random matrices Phys. Rev. E 82031135

[29] Khorunzhiy O, Kirsch W and Müller P 2006 Lifshitz tails for spectra of Erdös-Renyi random graphs Ann. Appl. Probab. 16 295-309 (arXiv:math-ph/0502054) 